\title{
The prognostic significance of thrombocytosis in ovarian cancer
}

\author{
Aneta Słabuszewska-Jóźwiak', Ewa Dmoch-Gajzlerska², Barbara Kozakiewicz², Grzegorz Jakiel' \\ ${ }^{1}$ The First Departament of Obstetrics and Gynecology Medical Centre of Postgraduate Education, Warsaw, Poland \\ ${ }^{2}$ Medical University of Warsaw, Poland
}

Słabuszewska-Jóźwiak A, Dmoch-Gajzlerska E, Kozakiewicz B, Jakiel G. The prognostic significance of thrombocytosis in ovarian cancer. Ann Agric Environ Med. 2015; 22(4): 731-735. doi: 10.5604/12321966.1185785

\begin{abstract}
Ovarian cancer makes up $25-30 \%$ of all cases of cancers of the female genital tract. It has the highest mortality rate of any condition in oncological gynaecology. Early diagnosis is associated with a favourable 5 -year survival prognosis. Many solid tumours have been detected with concomitant thrombocytosis. The tumour cell-induced platelet aggregation is a result of a direct integration of tumour cells with blood platelets. The aim of the present paper is an evaluation of platelet count as a prognostic parameter for ovarian cancer. Between 2000 - 2005, 349 patients with ovarian tumour (aged 12 - 88-years-old) underwent primary surgical treatment at a clinic. Ninety-seven patients with ovarian carcinomas underwent chemotherapy in the Oncology Centre. The control group comprised 252 women diagnosed with a histopathological lesion of mild intensity, whereas the cancer group constituted 97 women with a histopathologically-diagnosed malignant neoplasm. Thrombocytopaenia was assumed with a platelet count below $150 \mathrm{G} / \mathrm{L}$ and thrombocythaemia at $350 \mathrm{G} / \mathrm{L}$ and higher. Thrombocytosis often coincides with ascites and the cytoreduction decreases platelet count. There is a positive correlation between platelet count and tumour grading. Thrombocytosis was more frequently found in high grade tumours. There is also a positive correlation between platelet count and tumour stage according the International Federation of Gynaecology and Obstetrics (FIGO). Thrombocytosis was more frequently found in stage III and IV cancers. Patients with co-occurring thrombocytosis were found to have shorter survival periods and shorter time free from disease. This seems to give grounds for measuring platelet count before the primary surgical intervention, and suggests that the platelet count should be included in the panel of prognostic factors for patients with ovarian tumours.
\end{abstract}

\section{- Key words}

platelet count, thrombocytosis, ovarian cancer

\section{INTRODUCTION}

Ovarian cancer constitutes $25-30 \%$ of all malignant neoplasms of the female genital tract. It has the highest mortality of any condition in oncological gynaecology in Poland, Western Europe and the USA. In Poland in 2010, 3,587 new cases of ovarian cancer and 2,547 associated deaths were recorded, whereas in the USA in 2010,21,880 new cases of ovarian cancer were diagnosed and 13,850 associated deaths recorded.

Despite improved surgical techniques and the introduction of new methods of chemical treatment, the 5-year survival rate for the disease has not changed, remaining at $48.4 \%$ [1]. This is due to the fact that $75 \%$ of ovarian cancers are diagnosed during the late stage of progression. Survival depends on many factors, including stage of cancer progression, histological type, age of the patient, extent of surgery and subsequent treatment. Morbidity from ovarian cancer increases with age. Approximately $80-90 \%$ of cases appear after the 40th year. In Poland, diagnosis is most frequent in women aged 45-54 [2]. Among women under 30, approximately 3 per 100,000 fall ill, while above the age of 50 the risk increases more than ten-fold, with over 37 cases per 100,000 women recorded annually.

The majority of current diagnostic methods are not capable of diagnosing early, asymptomatic lesions in an ovary.

Address for correspondence: Aneta Słabuszewska-Jóźwiak, The First Departament of Obstetrics and Gynecology Medical Centre of Postgraduate Education, Warsaw, Poland, Pereca 2/410, 00-849 Warsaw, Poland

E-mail: as.jozwiak@op.pl

Received: 27 May 2013; accepted: 30 October 2013
Transvaginal ultrasongraphy, X-ray computed tomography, magnetic resonance imaging and the tumour marker values CA-125 and CEA are the only commonly available diagnostic methods for ovarian cancer, and have suboptimal specificity for ovarian cancer. As a result, there is ongoing research into new tumour markers which would allow an early diagnosis of ovarian cancer and its reoccurrence.

Blood platelets in neoplasm diseases show both quantitative and qualitative changes. Qualitative changes include: reduced activity of plasmin and plasminogen inhibitor activator, abnormal platelet aggregation, increased thromboxane and intensified production of thrombin caused by the abnormal presence of phosphatidylserine on the surface of a platelet membrane. The increase of blood platelet count above $5 \times 10^{5} /$ $\mathrm{ml}$ leads to an increased risk of thrombosis. In $30 \%$ of cases, thrombocythaemia is a reaction to tumour dissemination. The tumour cell-induced platelet aggregation is a result of the direct integration of tumour cells with blood platelets $[2,3]$. Among the factors involved in the integration are: thrombin, production of ADP and cysteine protease activity similar to cathepsin B, and a high concentration of the Von Willebrand factor.

Pinedo et al. hypothesised that blood platelets play an active part in the neoangiogenesis of a tumour [4]. This theory is based on the observation that thrombocytes are a rich source of pro- and anti-angiogenic factors and have the ability to combine with endothelium. Vascular Endothelial Growth Factor released from blood platelets stimulates the development of megakaryocytes and blood platelets, as well as neoangiogenesis of a tumour. 
Objective. The aim of the presented paper is evaluation of the platelet count as a prognostic parameter for ovarian cancer.

\section{MATERIALS AND METHOD}

The subjects were 349 patients aged between $18-88$, treated in the Professor W. Orłowski Clinic of Gynaecology and Obstetrics at Postgraduate Medical Education Centre in Warsaw between 2000-2005, for whom a platelet count was obtained one day before surgery.

The control group comprised 252 women diagnosed with a mild histopathological lesion, whereas the cancer group comprised 97 women with a histopathologically-diagnosed malignant neoplasm (Tab. 1). The cancer stage was assessed according to the FIGO system.

The cancer patients underwent cytoreduction surgery or a postponed cytoreduction surgery, followed by chemotherapy with platinum derivatives in the Maria Skłodowska-Curie Institute in the Warsaw Oncology Centre.

The platelet count for all patients wasmeasured inlaboratories in the above-mentioned centres. Thrombocytopaenia was assumed at $<150 \mathrm{G} / \mathrm{L}$ and thrombocythaemia at $\geq 350 \mathrm{G} / \mathrm{L}$.

Table 1. Histopathological diagnosis in the cancer group

\begin{tabular}{lcc}
\hline \multicolumn{1}{c}{ Histopathological diagnosis } & $\begin{array}{c}\text { No. of } \\
\text { cases }\end{array}$ & $\begin{array}{c}\text { Percentage } \\
\%\end{array}$ \\
Adenocarcinoma & 63 & 64.9484 \\
\hline Carcinoma clarocellulare & 10 & 10.3092 \\
\hline Carcinoma endometrioides, adenocarcinoma serosum & 1 & 1.03092 \\
\hline Carcinoma endometrioides & 13 & 13.4020 \\
\hline Cystadenocarcinoma papillarae of borderline malignancy & 5 & 5.1546 \\
\hline Cystadenofibroma papillarae of borderline malignancy & 2 & 2.0618 \\
\hline Cystadenoma mucinosum of borderline malignancy & 1 & 1.0309 \\
\hline Sertoli Leyding tumour & 2 & 2.0618 \\
\hline Total & 97 & 100
\end{tabular}

\section{RESULTS}

In the control group, the average platelet count before the surgical procedure was $237 \mathrm{G} / \mathrm{L}$, whereas in the cancer group it was $323.9 \mathrm{G} / \mathrm{L}$. The lowest platelet count in the control group was $76 \mathrm{G} / \mathrm{L}$ while in the cancer group it was $122 \mathrm{G} / \mathrm{L}$, this value occurring in a patient with stage $1 \mathrm{C}$ adenocarcinoma with high histological diversity. The maximum platelet count before the surgical procedure in the control group was $494 \mathrm{G} / \mathrm{L}$, and was found in a patient who received a postoperative diagnosis of an ovarian fibroid. In the cancer group, the maximum platelet count before the surgical procedure was $812 \mathrm{G} / \mathrm{L}$ in a patient with stage III adenocarcinoma with low histological diversity (Fig. 1).

Thrombocythaemia was diagnosed in 39 patients with ovarian cancer, while in the control group of patients with a mild ovarian tumour, thrombocythaemia was diagnosed in 17 patients.

Cytoreduction surgery. As in the majority of clinical trials we classed as optimal cytoreduction a surgery where we reduced macroscopic lesions to $<1 \mathrm{~cm}$ diameter. In 39 cases, of

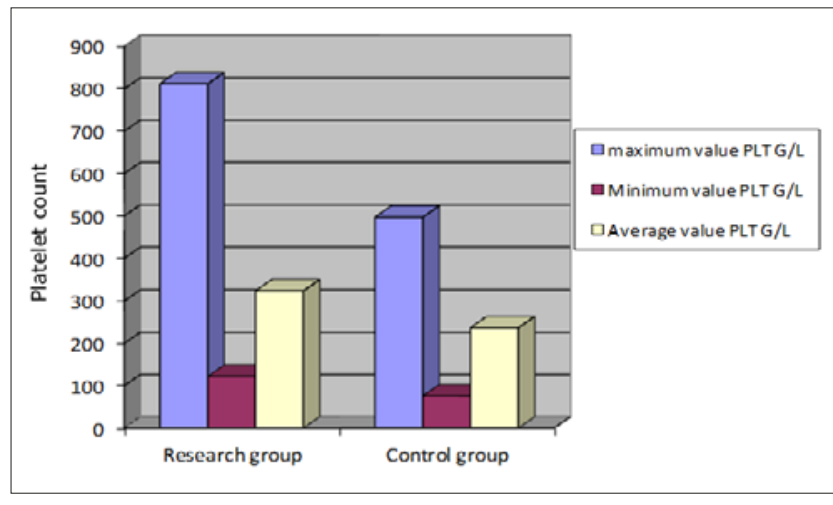

Figure 1. The platelet count in the research and control group

which 13 had a diagnosis of thrombocythaemia, the surgical procedure proved to be an optimal cytoreduction. In patients with a platelet count of $<350 \mathrm{G} / \mathrm{L}$, an optimal cytoreduction surgery was achieved in 41 of 57 cases (Fig. 2).

It should be stressed that there was a statistically significant difference between thrombocytothaemic cancer patients and non-thrombocytothaemic cancer patients in terms of optimal cytoreduction surgery; with an optimal cytoreduction procedure achieved more often in the patients with a normal $(<350 \mathrm{G} / \mathrm{L})$ platelet count $(p=0.00018)$.

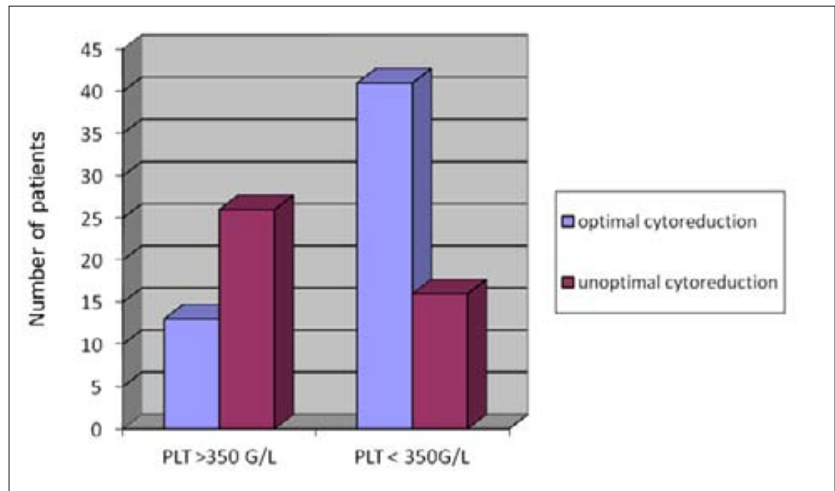

Figure 2. Cytoreduction in the cancer group: comparison of patients with a platelet count $>350 \mathrm{G} / \mathrm{L}$ and $<350 \mathrm{G} / \mathrm{L}$ ( $p=0.00018)$.

Ascites. Ascites was diagnosed in the presence of $>2000 \mathrm{ml}$ liquid in the peritoneal cavity. In 56 patients with ovarian cancer the presence of ascites was confirmed, in 32 of these women the platelet count was $>350 \mathrm{G} / \mathrm{L}$. In breast cancer patients with a normal platelet count $(<350 \mathrm{G} / \mathrm{L})$ ascites occurred only in 2 cases. These differences are statistically significant ( $\mathrm{p}=0.00034)$ (Fig. 3).

\section{Degree of histological diversity.}

In 29 cases, the ovarian tumours showed low histological diversity (G2); 13 cases showed high histological diversity (G1).

Cancer patients whose tumour showed a high or medium degree of tumour diversity were more likely to have thrombocythaemia $(p=0.0067)$.

\section{Clinical progression.}

Progression of disease was classified according to the FIGO system. Thrombocythaemia was present in 49 patients with stage III tumours $(p=0.0002)$. There were significant differences in platelet count between patients with tumours at stages I and III, and stage I and IV (Fig. 4). 


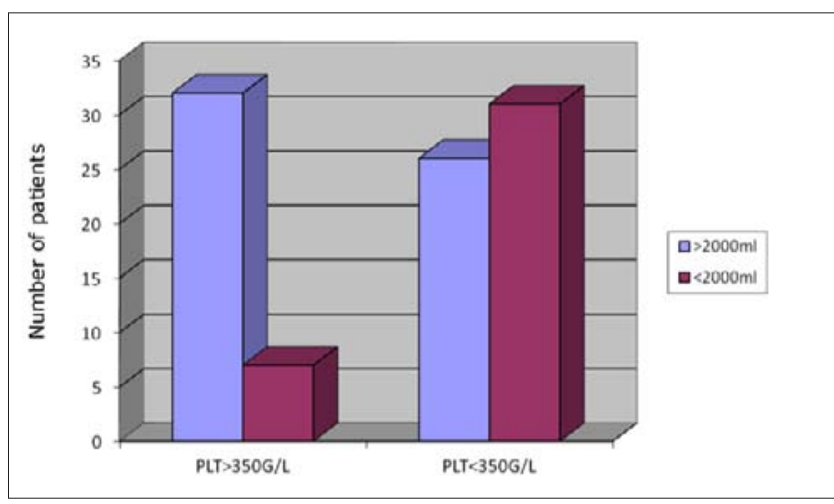

Figure 3. The comparison of ascites presence in the sick group with the platelets count above $350 \mathrm{G} / \mathrm{L}$ and $<350 \mathrm{G} / \mathrm{L}$

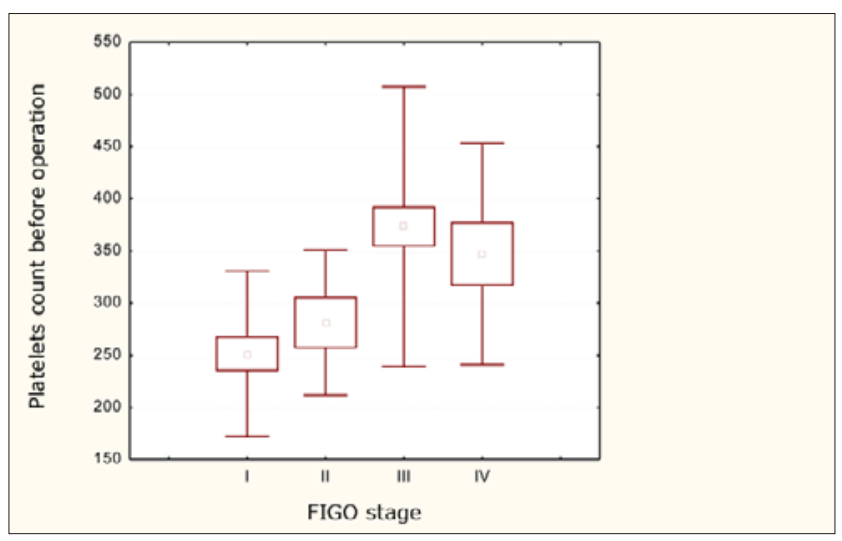

Figure 4. Relationship between disease stage and platelet count.

\section{Disease-free survival.}

We analysed whether platelet count before a surgical procedure is a predictor for ovarian cancer, by calculating the disease-free survival was period: the difference between the date of the first course of chemotherapy in the secondary line of neoplasm disease and the date of the sixth course of chemotherapy of the first line of neoplasm disease. A comparison was made between the length of the disease-free survival period for cancer patients with thrombocythaemia, and cancer patients with a normal platelet count (Fig. 5).

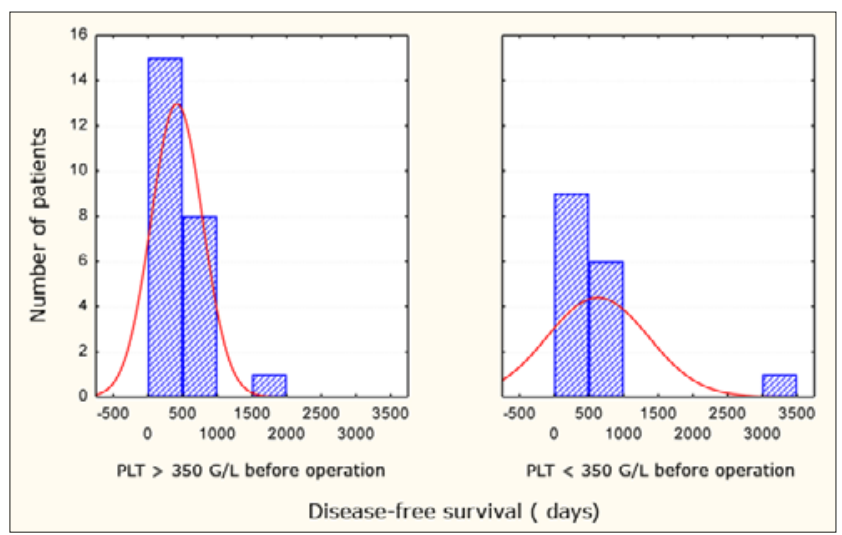

Figure 5. Comparison of distributions of disease-free survival in different groups.

The Mann-Whitney U test showed no differences in diseasefree survival between patients with thrombocythaemia and patients with a normal platelet count $(p=0.257)$. Analysis of occurrence time of the second line of neoplasm disease and its relationship with the platelet count showed that in the group with thrombocythaemia the second line of the disease occurred in 8 cases, while in the group with the platelet count of $<350 \mathrm{G} / \mathrm{L}$ it occurred in 31 cases. On this basis, a relationship between thrombocythaemia and the occurrence of the second-line of a disease was excluded $(p=0.4722)$.

\section{Total survival time (in days).}

The total survival time was assumed to be the number of days from the date of an ovarian cancer diagnosis to death, or to 6 September 2009 (last day of observations) for the patients who survived until this date.

The Mann-Whitney U test showed that the average survival period is shorter in the patient group with preoperative thrombocythaemia (mean survival=1579 days) than in the patient group with a platelet count of $<350 \mathrm{G} / \mathrm{L}$ (2178 days) $(p=0.0041)$ (Fig. 6). Patients with thrombocythaemia were 1.64 times less likely to achieve the mean survival time for the cancer group. On this basis, it can be asserted that a high platelet count is an unfavourable predictor for ovarian cancer.

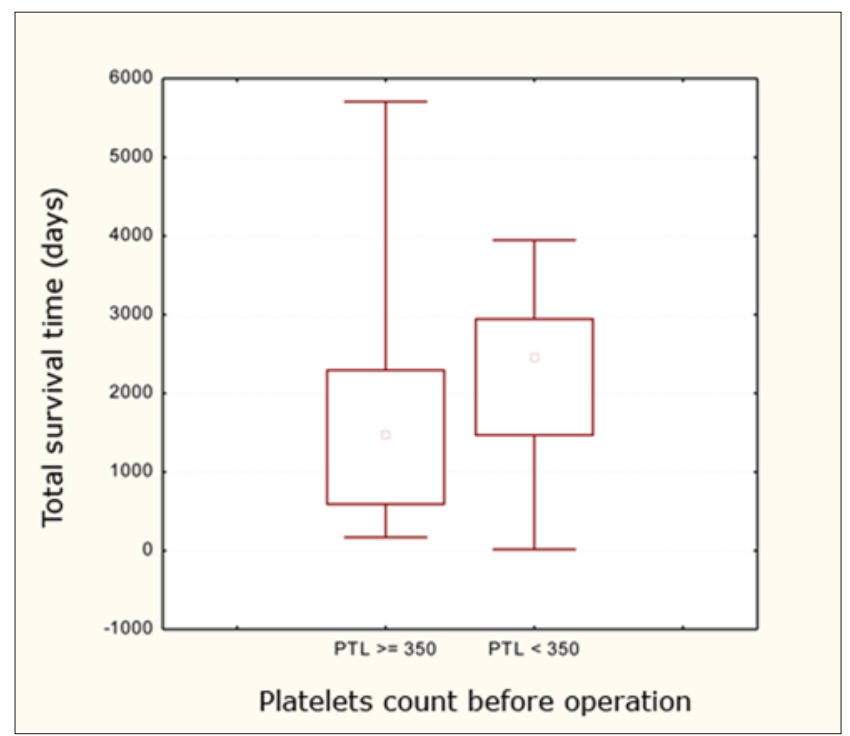

Figure 6. Relationship between platelet count before surgery and total survival time.

\section{DISCUSSION}

The increase in the concentration of blood platelets in malignant neoplasms was observed for the first time by Reiss in 1872. Currently, there are some reports which describe more frequent occurrences of an increased blood platelet count $(>400 \mathrm{G} / \mathrm{L})$ in ovarian cancer, in relation to non-malignant lesions of this organ. An increased platelet count is associated with tumours of the organs of the minor pelvis and beyond [5]. Increased thrombocytosis is found in 30-60\% of patients with non-gynaecological neoplasms, and in $10-30 \%$ of patients with neoplasms of the reproductive system including ovarian cancer, vulvar cancer [6], cervical cancer and endometrial cancer [7]. Many research papers have presented contradictory findings on the significance of thrombocytosis as a diagnostic and prognostic parameter in ovarian cancer.

The progression of neoplasm disease is related to hypercoagulability and an increased risk of thrombosis in the host organism. However, venous thromboembolism is often 
the first clinical symptom of a neoplasm. Trousseau (1872) was the first to observe the relationship between vein thrombosis and the presence of ovarian cancer. Since then many scientific papers have investigated the relationship between increased ovarian cancer morbidity and the progression of deep vein thrombosis (DVT) and pulmonary embolism (PE).

Homeostatic disorders in neoplasm diseases may result from the influence of neoplasm cells on the blood coagulation system and fibrinolysis, on endothelium and blood platelets. Tumour cells have the ability to produce and release their own pro-embolitic substances (cytokines, tissue factor, receptor for factor $\mathrm{V}$ ) which activate a coagulation cascade and have fibrinolytic characteristics (among others, tissue plasminogen activator; t-PA). Secreted cytokines may also contribute to metastasis by affecting vessels' endothelial cells, which start to attract neoplasm cells more strongly and facilitate their extravasation [8].

The mechanisms of blood coagulation are also connected to the response of the host to the presence of a tumour, and involve acute phase reaction and necrosis, abnormal protein metabolism and haemodynamic disorders, e.g. congested venous blood.

Morgan et al. found that among gynaecological neoplasms, ovarian cancer was the one most often accompanied by thrombosis [9]. According to Murchison [10], venous thromboembolism increases twofold the risk of ovarian cancer morbidity in individuals over 60 years of age. As a result, it is recommended that diagnostics in persons with idiopathic DVT/PE be extended to include these indicators as they may influence prognosis and treatment of patients with neoplasm disease. According to some reports, only $12 \%$ of patients with an ovarian tumour and venous thrombosis survive for one year [11], which supports the hypothesis that the coexistence of venous thrombosis and ovarian cancer worsens the prognosis. In the presented study, DVT of the lower limbs was diagnosed in $4(4.14 \%)$ patients in the cancer group. In 2 of these patients, the occurrence of thrombosis was preceded by an ovarian tumour diagnosis, 1 of these patients was neoplasm disease positive (breast cancer, tongue cancer). In the other 2 patients, thrombosis occurred during the third course of chemotherapy with platinum and paclitaxel. At the time their thrombosis was diagnosed, all these patients also had thrombocythaemia, ascites, and, postsurgery, and a late stage tumour. The average survival time in these patients was 389 days. On this basis, it can be concluded that the co-occurrence of thrombosis and ascites worsens the prognosis for ovarian cancer. This hypothesis is also confirmed by the work of other researchers. Sorensen [12] claimed that when cancer is diagnosed at the same time as the presence of thrombosis, or up to a year after a thrombosis episode, it is associated with a higher degree of neoplasm progression and worse prognosis. Similar conclusions were drawn by Rodrigues [13], who found thrombosis to be an independent prognostic predictor, correlating with the stage of clinical progression, and predicting reduced survival time for patients suffering from ovarian cancer. In another study, Sud et al. [14] confirmed the correlation between thrombosis and the existence of remote metastases 3 months after a diagnosis of neoplasm disease.

Gungor et al. analysed the cases of 292 women diagnosed with ovarian cancer who underwent a primary surgical procedure. In 124 (42.5\%) cases thrombocythaemia above $4.00 \times 10^{11} / \mathrm{L}$ was found. These patients showed significantly higher values of the preoperative marker CA-125, had a neoplasm at a later stage of clinical progression, and a lower degree of histological diversity. Thrombosis was found to be a significant negative predictor of survival of patients with ovarian epithelial cancer [15]. These results are in accordance with the observations in the presented study.

Menczer et al. conducted a similar study analysing 288 cases with ovarian cancer. They found that thrombocytosis co-occurred more frequently with ovarian cancer (70 patients with invasive cancer), than with its mild cysts or tumours with limited malignancy $(8.3 \%)$. However, they did not find a difference in the degree of thrombocytosis between malignant and non-malignant tumours. They also noted that the 5-year survival rate was lower in patients with an increased platelet concentration [16].

Zeimet et al. analysed the cases of 130 women with ovarian cancer, of whom 48 (37\%) were diagnosed at an early stage (stages I and II). They noted that thrombocythaemia was associated with more advanced disease, higher values of neoplasm marker CA-125, more ascites and a suboptimal surgical procedure (remaining fragments of tumour $>2 \mathrm{~cm}$ diameter). Nonetheless, the total survival time for patients was not correlated with thrombocythaemia [17].

Similar conclusions were presented by Li et al. in their paper [18]. These authors reported that patients with a higher preoperative platelet count showed a higher concentration of the antigen CA-125, more advanced clinical progression and lower tumour histological diversity (G3). Higher preoperative platelet count was also characteristic of cases with metastases to the lymph glands, a bigger volume of liquid in the abdominal cavity and a lower probability of conducting cytoreduction surgery. The authors compared the prognosis for patients with stage III and IV disease who also had thrombocytosis, and found that patients with thrombocythaemia and later stage disease were characterised by two-fold shorter disease-free survival time, and three-fold shorter total survival time [18].

Lee et al. (2011) analysed the cases of 179 women with ovarian cancer and found that 62 patients (34.6\%) had thrombocythaemia before the primary surgical procedure. These patients had higher values of the marker CA-125 and were more frequently diagnosed with ascites [19]. Unlike Li et al. [18] and Zeiment et al. [17], Lee et al. did not find significant differences in thrombocytosis when comparing stage III and IV stage patients with stage I and II patients. The main difference between these studies was in the proportion of patients at stage II and IV (63\% vs. $79.7 \%)$.

In the presented study, $50.3 \%$ of patients had stage II disease and $12.5 \%$ had stage IV disease; thrombocythaemia was associated with more advanced disease and a lower degree of tumour histological diversity. These differences were mainly observed in stage I patients, but were also true for stage II and IV patients ( $p=0.0002$ ) (R:55.520 and R: 53.750$)$. These results provide further support for the conclusion from previous studies, that thrombocythaemia is associated with a later stage of clinical progression.

Tumour aggression may be related to blood platelet count, as suggested by the significantly shorter mean total survival time $(p=0.0041)$ for patients with thrombocythaemia $(1,579$ days), compared to patients with a platelet count of $<350 \mathrm{G} / \mathrm{L}$ (2,178 days).

The alternative hypotheses that thrombocytosis is a reaction to the clinical progression of a tumour, or that it is an 
autonomous cause of the biological increase in the dynamics of a tumour, have not been fully investigated. However, there is evidence suggesting that platelets play a role in the creation of neoplasm metastases; neoplasm cells may directly activate platelets which, by covering the antigen determinants of neoplasm cell,s protect them against the immunological responses of a host. One may wonder at Demers's (2011) findings on the efficacy of chemotherapy for the treatments of breast cancer in mice with induced thrombocytopaenia. According to Demers, thrombocytopaenia, which influences the sealing of blood vessels, may contribute to the efficacy of chemotherapy [20].

It was also concluded from the cancer patient group in the current study that the platelet count depends on the degree of histological diversity. Differences in the platelets count are most marked between tumours with low and high degrees of histological diversity $(p=0.0012)$. Bozkurt et al. [21] found that a high platelet count in advanced ovarian cancer is associated with disease progression and tumour aggression. These conclusions were confirmed by Gungor et al., who found that in patients with a platelet count above $4.00 \times 10^{11} / \mathrm{L}$, clinical progression at diagnosis was more advanced, tumour histological diversity was lower, and survival time was shorter; this study also identified thrombocytosis as a negative prognostic factor in ovarian epithelium cancer [15].

Pinedo et al. proposed that blood platelets take an active part in the vascularisation of a tumour and the creation of neoplasm metastases [4]. Bozkurt et al. correlated platelet count, the results of a second-look surgery and the progression of disease in 38 patients with ovarian epithelium cancer (37 at FIGO stage III; 1 at stage IV) who underwent 6 courses of platinum-paclitaxel chemotherapy. Platelet count was assessed 5 days before the planned surgery. Analysis showed that platelet count was correlated with the stage of disease progression $\left(4.46 \pm 1.00 \times 10^{11} / \mathrm{L}\right.$ vs. $\left.3.71 \pm 9.4 \times 10^{11} / \mathrm{L}\right)$, disease progression and a positive result of second-look surgery; it was also found to be an important predictor of the presence of metastases [5]. In the cancer patient group in the currenr staudy, in only 8 patients did thrombocythaemia precede the occurrence of the second line of the disease, whereas in patients with the platelet count $<350 \mathrm{G} / \mathrm{L}$, disease progression was diagnosed in 31 cases. Consequently, on the basis of the obtained data, a relationship between thrombocythaemia and the occurrence of another line of disease $(p=0.4722)$ cannot be proved, possibly as a result of the small number of cases.

\section{CONCLUSIONS}

A higher platelet count was found in patients suffering from ovarian cancer, the platelet count was higher in patients with ascites, a lower degree of tumour histological diversity, and more advanced disease according to the FIGO system. Patients with a higher platelet count had a shorter mean survival time. On this basis, it is suggested that a platelet count before the primary surgical intervention be included in the panel of prognostic factors for patients with ovarian tumours.

\section{REFERENCES}

1. Markowska J, Markowska A. Epidemiologia i etiopatogeneza raka. In: Markowska J (ed.). Ginekologia Onkologiczna. Wroclaw, Urban \& Partner, 2006.p.805-6 (in Polish).

2. Wojtukiewicz MZ. Zakrzepy a nowotwory. In: Łopaciuk S (ed.) Zakrzepy i zatory. Warsaw, Wydawnictwo Lekarskie PZWL, Wyd. II, 2002.p.105-124 (in Polish).

3. Von Tempelhoff GF, Heilmann L, Hommel G, Schneider D, Niemann F, Zoller H. Hyperviscosity syndrome in patients with ovarian carcinoma. Cancer. 1998; 82: 1104-11.

4. Pinedo HM, Verheul HMW, D' Amato RJ, Folkman J. Involvement of platelets in tumor angiogenesis? Lancet. 1998; 352: 1775-1777.

5. Lavie O, Comerco G, Daras V, Bolger BS, Lopes A, Monaghan JM. Thrombocytosis in women with vulvar carcinoma. Gynecol Oncol. 1999; 72: 82-6.

6. Hefler L, Mayerhofer K,Leibman B, Obermair A, Reinthaller A, Kaintz $\mathrm{C}$, et al. Tumor anemia and thrombocytosis In patient with vulvar cancer. Tumor Biol. 2000; 21: 309-14.

7. Gucer F, Moser F, Tamussino K, Reich O, Haas J, Arikan G, et al. Thrombocytosis as a prognostic factor in endometrial carcinoma. Gynecol Oncol. 1998; 70: 210-214.

8. Honn KV, Tang DG, Chen YQ. Adhesion molecules and sitespecific metastates. Florance, Scientific Press, 1992.

9. Morgan MA, Iyengar TD, Napiorkowski BE, Rubin SC, Mikuta JJ. The clinical course of deep vein thrombosis in patients with gynecologic cancer. Gynecol Oncol. 2002; 84: 67-71.

10. Murchison JT, Wylie L, Stockton DL. Excess risk of cancer in patients with primary venous thromboembolism: a national, population-based cohort study. British J Cancer 2004; 91: 92-95.

11. Khorana AA, Francis CW, Culakova E, Lyman GH. Risk factors for chemotherapy-associated venous thromboembolism in a prospective observational study. Cancer. 2005; 15(104): 2822-2829.

12. Sorensen, HT, Mellemkjaer L, Olesn JH, Baron JA. Prognosis of cancers associated with venous thromboembolism. N Engl J Med. 2000; 343:1846-1850.

13. Rodriguez AO, Wun T, Chew H. Venous thromboembolism in ovarian cancer. Gynecol Oncol. 2007; 105: 784-790.

14. Sud R, Khorana AA, Cancer-associated thrombosis: risk factors, candidate biomarkers and risk model. Thrombosis Research. 2009; 123, S18-S21.

15. Gungor T, Kanat-Pektas M,Sucak A, Mollamahmutoglu C. The role of thrombocytosis in prognostic evaluation of epithelial ovarian tumors. Arch Gynecol Obstet. 2009; 279: 53-56.

16. Menczer J, Schejter E, Geva D, Ginath S, Zakut H. Ovarian carcinoma associated thrombocytosis: correlation with prognostic factors and with survival. Eur J Gynaecol Oncol. 1998; 19: 82-84.

17. Zeimet AG, Marth C, Muller-Holzner E, Daxenbichler G, Depunt O. Significance of thrombocytosis in patients with epithelial ovarian cancer. Am J Obstet Gynecol. 1994; 170: 549-554.

18. Li AJ, Madden AC, Cass I, Leuchter RS, Lagasse LD, Karlan BY. The prognostic significance of thrombocytosis in epithelial ovarian carcinoma. Gynecol Oncol. 2004; 92(1): 211-214.

19. Lee M, Kim SW, Nam EJ, Yim GW, Kim S, Kim YT. The impact of pretreatment thrombocytosis and persistent thrombocytosis after adjuvant chemotherapy in patients with advanced epithelial ovarian cancer. Gynaecol Oncol. 2011; 122: 238-241.

20. Demers M, Ho-TinNoe B, Schatzberg D, Yang JJ, Wagner DD. Increased efficancy of breast cancer chemotherapy in thrombocytopenic mice. Cancer Res. 2011; 71(5): 1540-1509.

21. Bozkurt N, Yuce K, Basaran M, Kose F, Ayhan A. Correlation of platelet count with second look laparotomy results and disease progression in patients with advanced epithelial ovarian cancer. Obstet Ggynecol. 2004; 103: 82-85. 